\title{
Investigating Optimization Strategies that Determine Performance of CMS Websites in Organizations
}

\author{
Samuel K. Shitote ${ }^{1}$, Dr. Kelvin. Omieno ${ }^{2}$, Dr. Jaspser. Ondulo ${ }^{3}$ \\ Student, Information Technology, Masinde Muliro University of Science and Technology, Kakamega, Kenya ${ }^{1}$ \\ Lecturer, Computer Science, Masinde Muliro University of Science and Technology, Kakamega, Kenya ${ }^{2,3}$
}

\begin{abstract}
Web Content Management Systems (WCMS) have evolved over years from very primitive systems to complex and robust intelligible systems. These systems provide the ability to maintain and navigate the structure of a website and allow the developers to design websites more easily, hence making content publishing on the internet fast and easy. Due to the complexity of CMS, most corporate websites tend to experience performance downside. Studies reveal that a lot is being done to improve websites performance. Several web optimization techniques have been adopted to reduce pageloadtime and much has been achieved but as the web content keeps on growing, performance is lowering. This study tries to address the CMS website performance gaps by providing strategies that can achieve better performance.. The study employs experimental research design. Joomla, Wordpress and Drupal CMS were used in the experiment to represent the most popular CMS used in organizations. The experiments were done in Moi university labs. Optimization process was achieved using GTMetrix and GiftOfSpeed online tools. The findings were that: Image optimization, CSS/JS \& HTML Minificaition, Caching, mobile optimization had significant impact on CMS website performance. A combination of these techniques results to web optimization strategies that will improve CMS website performance
\end{abstract}

Keywords: Content Management Systems, Drupal, Joomla, Wordpres, Performance

\section{INTRODUCTION}

In this digital age, organizations are adopting various modes of communication to disseminate information to their audience; their main goal being to reach to potential clients and also to maintain a strong relationship with those already existing. Winning the heart of a client is a big challenge, due to high level of competition among various organizations. To achieve this goal most organizations are now moving away from the analogue form of communication to digital so as to provide real-time information to its clients. Universities like other organizations have a primary goal of communicating academic information and to offer training in modern technology.

The consumers of academic information have an expectation of receiving quality information since universities are so vast in technological know-how. Information has to undergo a process for it to become meaningful to the user. Information and content are terms oftenly used as synonyms, but which has precise meaning that distinguishes one from the other. Information is defined as data that has been processed into a form that is meaningful to the recipient [1] while content is a suite of structured data that a computer can organize in a system for the collection, management and publication[2]. The authors further states that information becomes content when it is used for one or more purposes. Its value is the amount of its primary form, application, uniqueness, usability and significance [2].

With the rise in demand of online content, there has been a parallel rise in the importance of efficiency in all the procedures from information gathering and sorting to information dissemination, filtration and security. These online content consumers are not just looking for any content but valuable content. Content is said to be valuable when it is accurate, valid, reliable, timely, relevant and complete [3]. The need to manage content and information found an initial answer in knowledge and content management systems. Knowledge and content management systems provide the ability to maintain and navigate the structure of a website by the users and allow the developer to develop web applications more easily hence making content publishing on the internet fast and easy.

The complexity of content management systems structures provide a great advantage in managing content, however can also compromise on website performance. Studies have been done around this subject in a view to improve website performance; however more still need to be done. This study investigated web optimization strategies that could help improve website performance in organizations. 


\section{KEY CONCEPTS}

Content management system is a computer application that supports the creation, modification and publication of digital content using a common user interface and integrated multi-user collaborative environment. Through the use of CMS universities can now reach out to students, staff, supplier, policy makers e.t.c and share their expertise as need may arise.

The primary goal of any website owner is to increase web visibility of their websites in search engines in order to grow the sphere of his clientele. Most searchers use the results that appear on the first page in search engines without going further to the second or third results page [4].

A. Benefits of content management systems: According Gizatu \& Dino(2015) the biggest advantage of using Content Management system is having a structured platform for content publishing. Since, CMS uses standard MVC (Model View Controller) base framework, the content will be managed in hierarchical and structured way. Some of the benefits include: improved efficiencies, streamlined security, enhanced document management and automated business processes [4].

B. The most popular content management system used in organization: The study focused on the three most popular content management systems used in managing web content in organizations and these include Joomla, Wordpress and Drupal.

(a) Joomla: This is one of the most powerful open source CMS used for publishing and creating highly interactive multi-language websites in short time like online communities, media, portals, blogs and E-commerce applications [4]. The following are extensions available for Joomla: components, modules, plugins, templates, and languages and each of these extensions deals with unique functionalities. Some of the most distinguished clients of Joomla include: Linux Corporation, Harvard university graduate school, green maven, playshakespeare etc.

(b) WordPress CMS: WordPress Content Management System is one of many PHP/MySQL content management systems that allow content editors to use a web interface to maintain their sites instead of editing and uploading HTML files to a server. WordPress won the overall Best Open Source CMS Awards, WordPress is still extensively considered as a blogging tool [5]. Some of the distinguished clients of WordPress include: tmagazine, policalticker etc.

(c) Drupal: Drupal is a free open source software package that enables users to easily publish, manage, control and organize content on a website and was developed in 2001. Drupal is easy to use, based on modules, extensible and standard-based content management system. Drupal modules and power pages offer much functionality such as blogs, picture galleries, extendible content, forums, community portals and corporate ecommerce sites. Some of the most distinguished clients of Drupal CMS include the websites for: Whitehouse, Ubuntu, London government, University of Nairobi among others.

\section{RELATED STUDIES}

Considerable studies have been done in the areas of website performance, web application optimization and content management systems. However little has been done on optimization of CMS websites to enhance performance. Content management systems have become common as most web developer shift to MVC technology. So far we have hundreds of CMSs on the market and users may not know which one to use and also how to optimize and get optimal performance. The following works formed the basis of this study:

Vihervaara etal. (2016) researched on Performance Gains from Web Performance Optimization. The study presented the results related to an optimization work where all the resources of the website were optimized[6]. The results obtained were very significant. The download size of the front page was reduced by a total of about 80 percent and the downloading time about 60 percent. The element of reducing page size for better performance was a contribution to this research work. However the study focused only on a single website referred to as ForeAmmatti, which offers information in respect of the labour market of Finland. Various CMSs have different optimization strategies which need to be looked into. Souer etal.(2008) researched on a Framework for Web Content Management System Operations and Maintenance [7]. The study presented a process framework for Web Content Management Systems. The researchers used fragments from existing models and combined them into a model. The framework consists of a set of guidelines and descriptions process separated over three managerial levels: strategic, tactical and operation. This research informed the researcher on developing a WCMS framework for organizations. However it did not address the issues of performance of CMS for developing websites. 
Quadri(2011) in his research titled: Developing, Managing and Maintaining Web Applications with Content Management Systems: Drupal and Joomla as case study, analysed how to develop different web applications with desired functionalities using Drupal and Joomla content management systems[8]. From this study, the researcher learnt how and where to add functionality in a Drupal and Joomla powered web application. That CMS software help organizations to increase efficiency and information outreach. The author again notes that, Drupal and Joomla are reliable, flexible, stable, secure, modular, supportive, extensible and less expensive. They can be used for all types of websites and web applications by individual or organizations. Therefore the study focused more on the functionality of the two CMS (Drupal and Joomla) but never addressed optimization component. Pyrzynski etal.(n.d.) in their study titled: Improving Content Management System performance. The authors established that after migrating Geoffresh.com from html to CMS (Joomla) the website became slow and they had to find out how to improve the performance [9]. Using experiments, they investigated XHTML and CSS validation as the major contributor to website performance or underperformance. They found out also that server performance affects the performance of websites. However, their research used only one CMS Joomla and did not consider investigating the impact of content (images) and other forms of optimization techniques on the CMS website performance.

Shailesh \& Suresh (2017) conducted a survey of state of the art tools, techniques, methodologies of various aspects of web performance optimization [10]. They elaborated on key aspects of pro-active quality in web applications and on various techniques related to Web Performance Optimization (WPO) such as image compression, monitoring and testing. The study detailed various web performance optimization techniques such as caching, performance design checklist, prefetching, performance testing, performance monitoring. The study informed the researcher on optimization techniques for website performance. But the study used only a survey research design to carry out the study.

Gomez Inc (2011) carried out a research titled: Why Web Performance Matters: Is Your Site Driving Customers Away? The study established that an average online shopper expects pages to load in two seconds or less, down from four seconds in 2006; after three seconds, up to $40 \%$ will abandon the site. The studies reveal that lack of visitor loyalty. By analysing page abandonment data across more than 150 websites and 150 million page views, the researcher found that an increase in page response time from 2 to 10 seconds increased page abandonment rates by $38 \%$. Finally the researcher found out that poor availability and page-load times have an immediate — and negative impact on customer experience. But with improving performance lead to more favourable business outcomes. He further compared the performance of optimized and un-optimized website and established that optimized website led to improved performance [11]. This study informed the researcher that there is a relationship between web performance and customer traffic on any given organizational website.

\section{EXISTING WEB OPTIMIZATION TECHNIQUES}

These are techniques that when applied will contribute to improved performance of websites. Based on the existing works, a number of authors have written on the subject, however more needs to be done on the same. This is because of the emerging trends in knowledge management systems. Infosys (n.d.) discusses the following performance gains of website optimization strategies [12]:

(i) Merge all the JS and CSS files: This technique minimizes the total number of resource requests, the lower the requests the better the performance.

(ii) Minify the JS and CSS files and the HTML content: Reduced size would minimize the response time. On an average there will be $30 \%$ reduction in the file size for JS and CSS files and about $20 \%$ size reduction in page size. The reduction of file size and page size will contribute the site to loading faster.

(iii) Place all external JavaScript links at the bottom and all CSS file links in the head element: As JS files are at the bottom of the page, it will not block serial load of the page. This would decrease the perceived load time for the user. Many times 3rd scripts related to analytics and other has the potential to slow down the entire site[12].

(iv) Assets Optimization: Use CSS sprites and lossless compression or PNG files. This will results in multi-fold benefits such as reducing the number or resource requests and reduced image size. Image compression would reduce the size by approximately $25 \%$ hence improving the overall CMS performance.

(v) Compression: Enable gzip compression for HTTP traffic. Absence of device specific site and mobile accelerator would render the page slow in mobile devices 
Vol. 7, Issue 10, October 2018

\section{METHODOLOGY}

The researcher investigated existing works on factors that determine websites performance. This study employed experimental research design. Experimental design was used to determine if a specific treatment on the experimental variable influences an outcome.

A. Experimental procedure: A sample of WordPress, Joomla and Drupal CMS websites were installed and hosted in Moi University servers. The researcher populated the three websites with similar type and size of content. The performance of the websites at default state (before optimization) was determined using GTmetrix online tool and readings recorded accordingly. Optimization procedures were carried out step by step as the readings were recorded after every strategy was applied. The process was repeated for different scenarios and results recorded.

B Experimental Environment: Experimental environment is where experiments for performance tests are executed with all the tools and hardware. According to Ramler \& Gmeiner(2014) test environment is a critical part of a project's software test infrastructure. Appropriate test environments are a prerequisite for effective test automation and can show a substantial impact on testability, defect detection, and overall test costs[13]. This study test was done in Moi University labs and sample tests hosted within the Moi University servers. The researcher used a HP EliteBook 8460 laptop to carry out experiments. The following architecture formed the basis of the experiments:

\section{(a) Laptop specification}

HP EliteBook 8460

Intel(R) Core(TM)i5-2520M CPU@ 2.50GHz, 2501 Mhz, 2 Core(s), 4 Logical Processor(s)

Physical Memory (RAM) 4.0 GB

System type. X64 based-PC

Operating system - Microsoft Windows 10Pro edition

Browser - Mozilla Firefox v.56

\section{(b) Hosting Server specifications}

\section{Name: Intel(R) Xeon(R) CPU X5450@3.00GHz}

Version: 6.7.6

Size: $3 \mathrm{GHz}$

Width: 64 bits

C. Experimental Tools: This section discusses various tools the researcher adopted for data collection. In this study the researcher used the following web application testing tools to determine the performance of the CMSs: Builtwith, GTMetrix and GiftOfSpeed web performance online tools were used.

D. Study area: The study area included all universities in Kenya, while Moi University labs were used to carry out the experiments. Kenya has a total of 48 chartered universities. Using BuiltWith online testing tools the researcher established that out of the 48 universities, 40 used either Joomla, Wordpress or Drupal CMSs to develop their websites. Table 4.1 below shows the results.

Table 1: CMS Popularly Used in Universities In Kenya

\begin{tabular}{|l|l|l|}
\hline CMS & Frequency & Percentages \\
\hline Wordpress & 9 & $18.75 \%$ \\
\hline Joomla & 24 & $50 \%$ \\
\hline Drupal & 7 & $14.58 \%$ \\
\hline Others & 8 & $16.67 \%$ \\
\hline Total & $\mathbf{4 8}$ & $\mathbf{1 0 0 \%}$ \\
\hline
\end{tabular}

E. Tools used for Testing Web performance: GTMetrix and GiftOfSpeed online tools were used to test the performance of the sample CMS websites. 
Vol. 7, Issue 10, October 2018

(i) GTMetrixs: This tool summarizes the web page performance based on key indicators of pageload speed. It analyzes the page with google page speed and yahoo, yslow rulesets. Gets page load time. Total Page Size and Total number of requests. Compares the page's performance relative to the average of all sites analyzed on GTmetrixs and keeps track of page performance with scheduled monitoring and visualize it with interactive graphs. It alerts the user when the page performs poorly. It analyzes the page load of a site from 28 servers in 7 different regions around the world. The tool also optimizes website for mobile users and ensure a fast page loadtime on all devices.

(ii) GiftOfSpeed: GiftOfSpeed has tools to compress PNG and JPEG images separately. It applies multiple image compression techniques to minimize the file sizes to lowest size possible. It allows one to resize the images for a greater performance online. Some of the tools that constitute GiftOfSpeed are: Gzip compression, CSS delivery, Browser caching, Lazy load images, Optimize images, make HTTS fewer requests and use less javascript.

\section{EXPERIMENTAL RESULTS}

Table 3 Performance of Joomla, Wordpress and Drupal CMS at Default Settings

\begin{tabular}{|l|l|l|l|}
\hline & Joomla & Wordpress & Drupal \\
\hline PageLoadTime & $12.8 \mathrm{~s}$ & $5.7 \mathrm{~s}$ & $4.9 \mathrm{~s}$ \\
\hline Total Page Size & $11.8 \mathrm{mb}$ & $941 \mathrm{~KB}$ & $1.69 \mathrm{mb}$ \\
\hline No. of Requests & 36 & 29 & 29 \\
\hline PageSpeedGrade & $\mathrm{F}(20 \%)$ & $\mathrm{A}(91 \%)$ & $\mathrm{B}(89 \%)$ \\
\hline YSlow grade & $\mathrm{C}(70 \%)$ & $\mathrm{C}(73 \%)$ & $\mathrm{C}(71 \%)$ \\
\hline
\end{tabular}

Based on Table 3 above Drupal website took the least time to load followed by WordPress then Joomla websites. Drupal and Wordpress performed better than Joomla at the default settings. The researcher observed that even after uploading the same size of content on the three CMSs, Wordpress and Drupal automatically optimized the page size to $941 \mathrm{~kb}$ and $1,69 \mathrm{mb}$ respectively. Joomla maintained the page size of $11.8 \mathrm{mb}$. Pagesize impacts on the no of requests made hence the performance of CMS.

Table 4 CMS Performance During Optimization Process

\begin{tabular}{|c|c|c|c|c|c|c|}
\hline & $\begin{array}{l}\text { Before } \\
\text { optimization }\end{array}$ & $\begin{array}{l}\text { Image } \\
\text { optimization }\end{array}$ & $\begin{array}{l}\text { Image } \\
\text { optimization } \\
+ \\
\text { Cache } \\
\text { Optimization }\end{array}$ & $\begin{array}{l}\text { Image } \\
\text { optimization } \\
+ \\
\text { Cache } \\
\text { Optimization } \\
+ \\
\text { JS/CSS } \\
\text { Minification }\end{array}$ & $\begin{array}{l}\text { Image } \\
\text { optimization } \\
+ \\
\text { Cache } \\
\text { Optimization } \\
+ \\
\text { JS/CSS } \\
\text { Minification } \\
+ \\
\text { HTML } \\
\text { minification }\end{array}$ & $\begin{array}{l}\text { Image } \\
\text { optimization } \\
+ \\
\text { Cache } \\
\text { Optimization } \\
+ \\
\text { JS/CSS } \\
\text { Minification } \\
+ \\
\text { HTML } \\
\text { minification } \\
+ \\
\text { Mobile } \\
\text { optimization }\end{array}$ \\
\hline \multicolumn{7}{|c|}{ JOOMLA CMS } \\
\hline PageLoadTime & $12.8 \mathrm{~s}$ & $3.2 \mathrm{~s}$ & $3.1 \mathrm{~s}$ & $2.6 \mathrm{~s}$ & $2.1 \mathrm{~s}$ & $1.5 \mathrm{~s}$ \\
\hline Total Page Size & $11.8 \mathrm{mb}$ & $0.97 \mathrm{mb}$ & $0.97 \mathrm{mb}$ & $497 \mathrm{~kb}$ & $407 \mathrm{~kb}$ & $407 \mathrm{~kb}$ \\
\hline No. of Requests & 36 & 36 & 36 & 22 & 20 & 20 \\
\hline PageSpeedGrade & $\mathrm{F}(0 \%)$ & $\mathrm{B}(80 \%)$ & $\mathrm{B}(80 \%)$ & $\mathrm{B}(89 \%)$ & $\mathrm{A}(91 \%)$ & $\mathrm{A}(93 \%)$ \\
\hline YSlow grade & $\mathrm{C}(70 \%)$ & $\mathrm{C}(70 \%)$ & $\mathrm{C}(70 \%)$ & $\mathrm{C}(76 \%)$ & $\mathrm{B}(80 \%)$ & $\mathrm{B}(86 \%)$ \\
\hline \multicolumn{7}{|c|}{ WORDPRESS CMS } \\
\hline PageLoadTime & $5.7 \mathrm{~s}$ & $4.2 \mathrm{~s}$ & $4.0 \mathrm{~s}$ & $3.4 \mathrm{~s}$ & $2.3 \mathrm{~s}$ & $1.8 \mathrm{~s}$ \\
\hline Total Page Size & $941 \mathrm{~kb}$ & $911 \mathrm{~kb}$ & $911 \mathrm{~kb}$ & $801 \mathrm{~kb}$ & $767 \mathrm{~kb}$ & $767 \mathrm{~kb}$ \\
\hline No. of Requests & 29 & 27 & 26 & 21 & 19 & 19 \\
\hline PageSpeedGrade & $\mathrm{A}(91 \%)$ & $\mathrm{A}(95 \%)$ & $\mathrm{A}(95 \%)$ & $\mathrm{A}(97 \%)$ & $\mathrm{A}(99 \%)$ & $\mathrm{A}(100 \%)$ \\
\hline YSlow grade & $\mathrm{C}(73 \%)$ & $\mathrm{C}(74 \%)$ & $\mathrm{C}(76 \%)$ & $\mathrm{C}(78 \%)$ & $\mathrm{B}(81 \%)$ & $\mathrm{B}(81 \%)$ \\
\hline
\end{tabular}


Vol. 7, Issue 10, October 2018

\begin{tabular}{|l|l|l|l|l|l|l|}
\hline \multicolumn{9}{|c|}{ DRUPAL CMS } \\
\hline PageLoadTime & $4.9 \mathrm{~s}$ & $4.7 \mathrm{~s}$ & $4.4 \mathrm{~s}$ & $3.1 \mathrm{~s}$ & $2.8 \mathrm{~s}$ & $1.6 \mathrm{~s}$ \\
\hline Total Page Size & $1.69 \mathrm{mb}$ & $1.1 \mathrm{mb}$ & $1.1 \mathrm{mb}$ & $937 \mathrm{mb}$ & $878 \mathrm{mb}$ & $878 \mathrm{mb}$ \\
\hline No. of Requests & 29 & 28 & 22 & 21 & 19 & 19 \\
\hline PageSpeedGrade & $\mathrm{B}(89 \%)$ & $\mathrm{B}(89 \%)$ & $\mathrm{A}(91 \%)$ & $\mathrm{A}(94 \%)$ & $\mathrm{A}(96 \%)$ & $\mathrm{A}(97 \%)$ \\
\hline YSlow grade & $\mathrm{C}(71 \%)$ & $\mathrm{C}(71 \%)$ & $\mathrm{C}(71 \%)$ & $\mathrm{C}(77 \%)$ & $\mathrm{B}(81 \%)$ & $\mathrm{B}(88 \%)$ \\
\hline
\end{tabular}

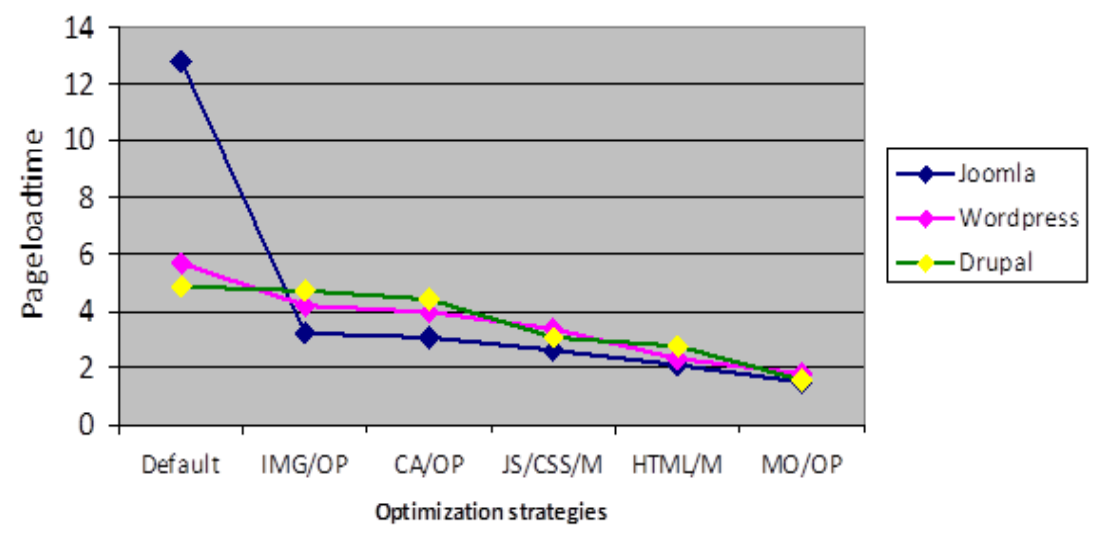

Fig. 1 A graphical representation of performance improvement of Joomla Wordpress and Drupal CMSs

Figure 1 above is a graphical representation showing the impact of optimization strategies on CMS performance. The following strategies showed significant impact on performance: Image optimization (IMG/OP), Cache optimization (CA/OP), JavaScript; Cascading Style Sheets (JS/CSS) Minification, Hypertext Markup Language (HTML) minification and mobile optimization.

\section{CONCLUSION}

The researcher achieved the objective of the study thus to determine optimization strategies that improve CMS websites performance; the literature review also revealed various optimizations techniques and from experiments that were performed the researcher found out that: Image optimization, Cache optimization, JS/CSS/HTML minification and mobile optimization had significant impact on CMS websites performance.

\section{REFERENCES}

[1]. C. Zins, "Conceptual Approaches for Defining Data , Information ,” vol. 58, no. January, pp. 479-493, 2007.

[2]. C. Benevolo and S. Negri, "Evaluation of Content Management Systems (CMS): a Supply Analysis,” Inf. Technol. Eval., vol. 10, no. 1, p. 14, 2007.

[3]. T. R. D. Council, “Three Rivers District Council Data Quality Strategy,” no. March, 2008.

[4]. L. Yu, "A Web of Data: Toward the Idea of the Semantic Web," in A Developer's Guide to the Semantic Web, Berlin, Heidelberg: Springer Berlin Heidelberg, 2014, pp. 3-21.

[5]. G. R. Marczyk, D. DeMatteo, and D. Festinger, Essentials of Research Design and Methodology, vol. Essentials. 2010.

[6]. J. Vihervaara, P. Loula, and T. Tuominen, "Performance Gains from Web Performance Optimization," vol. 1, no. Webist, pp. 188-193, 2016.

[7]. J. Souer, J. Versendaal, and H. Utrecht, "A Framework for Web Content Management System Operations and Maintenance .," no. JANUARY, 2008.

[8]. S. A. Quadri, "Developing, Managing and Maintaining Web Applications with Content Management Systems : Drupal and Joomla as case study .," 2011.

[9]. G. Pyrzynski, T. Park, and M. D. Theys, "Improving Content Management System Performance and the Web," no. Mc 152.

[10]. K. S. Shailesh and P. V Suresh, "An Analysis of Techniques and Quality Assessment for Web Performance Optimization,” Indian J. Comput. Sci. Eng., vol. 8, no. 2, pp. 61-69, 2017.

[11]. Gomez Inc, "Why Web Performance Matters : Is Your Site Driving Customers Away?,” pp. 1-8, 2011.

[12]. L. Infosys, "WHITE PAPER Presentation tier performance optimization."

[13]. R. Ramler and J. Gmeiner, "Practical Challenges in Test Environment Management," in 2014 IEEE Seventh International Conference on Software Testing, Verification and Validation Workshops, 2014, pp. 358-359. 